\title{
MODELO PARA LA CONSTRUCCIÓN COLECTIVA DE CONOCIMIENTO PARA LA RURALIDAD
}

\author{
MODEL OF COLLECTIVE CONSTRUCTION \\ OF KNOWLEDGE FOR RURALITY
}

${ }^{1}$ Deyser Gutiérrez A.
2 Mary Ortega G.
${ }^{3}$ Cesar Henao V.
${ }^{4}$ Carlos Torres V.
${ }^{5}$ Jonathan Sánchez G.

${ }^{1}$ Magister en Tecnología de la Información. UNAD. Medellín. Colombia. UNAD. Medellín. Colombia.

${ }^{2}$ Magister internacional en Dietética y Nutrición UNAD. Medellín. Colombia. UNAD. Medellín. Colombia.

${ }_{3}^{3}$ Magister en Entornos Virtuales de Aprendizaje.CUA. Medellín. Colombia. CUA. Medellín. Colombia.

${ }^{4}$ Especialista en Gestión de Infraestructura Tecnológica IUE. Envigado. Colombia. IUE. Envigado. Colombia.

${ }^{5}$ Magister en Software libre ITM. Medellín. Colombia. ITM. Medellín. Colombia.

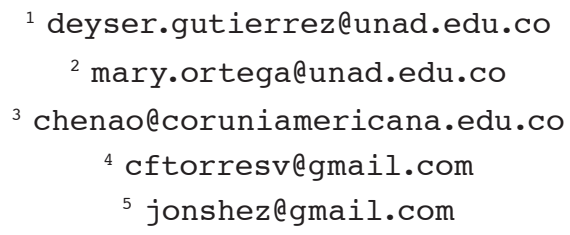

\section{RESUMEN}

El diseño de un modelo para la construcción colectiva para la ruralidad, tiene como objetivo visibilizar la pertinencia y calidad de la educación en los diferentes niveles, con la participación de actores sociales especializados y no especializados, dando cuenta de los pasos que se siguieron para elaborar un modelo colaborativo de Educación Superior Rural del Nordeste de Antioquia, con la participación de instituciones de educación superior de la región, que se unen para desarrollar, en la primera fase un diplomado, donde se apropia de las definiciones de ruralidad y conceptúa la pertinencia de modelos educativos; en la segunda fase, se desarrollan cursos cortos con la implementación de las tendencias, aproximación al contexto sociocultural que van a propiciar el modelo y adaptabilidad; interrelacionado la cultura, desarrollo y pertinencia en contexto, riqueza de conductas con una metodología exploratoria (cualitativa), con la finalidad de mejorar condiciones de 
asequibilidad, cobertura y calidad educativa, desde el abanico de realidades sociales. En la tercera fase se construye un modelo colaborativo basado en módulos en el marco de un currículo flexible y una alternativa sociocultural que propende por la reconstrucción del tejido social. En conclusión, el modelo para la construcción colectiva de conocimiento para la ruralidad se diseña colectivamente proponiendo el currículo flexible, didácticas creativas e innovadoras con prospectiva transformación del contexto sociocultural.

Palabras clave: Calidad educativa, Desarrollo de territorios, Educación Superior, Etnografía, Modelo colaborativo, Ruralidad.

\section{ABSTRACT}

The design of a model for collective construction for rurality aims to make visible the relevance and quality of education at different level. This includes the participation of specialized and nonspecialized social actors, giving an account of the steps followed to develop a collaborative model of rural higher education of the Northeast of Antioquia. In addition, it includes higher education institutions in the region, which come together to develop, in the first phase, a diploma course, where appropriate, the definitions and conceptualize the relevance of educational models. In the second phase, short courses are developed with the implementation of trends, and approach to the sociocultural context that will lead to the model and adaptability. Interrelating culture, development and significance in context, wealth of behaviors with an exploratory methodology (qualitative), with the aim of improving conditions of affordability, coverage and educational quality, from the range of social realities. In the third phase, a collaborative model based on modules is built within the framework of a flexible curriculum and sociocultural alternative that tends towards the reconstruction of social fabric. In conclusion, the model for the collective construction of knowledge for rurality is designed collectively proposing flexible curricula, creative and innovative didactic with prospective transformation of sociocultural context.

Keywords: Collaborative model, Educational quality, Ethnography, Higher education, Rural education, Territorial development

\section{INTRODUCCIÓN}

El modelo para la construcción colectiva de conocimiento para la ruralidad hace referencia a la articulación de un grupo de universidades antioqueñas, que propenden por el desarrollo de los territorios del nordeste antioqueño, mediante el reconocimiento del saber y la implementación del conocimiento, acorde con las necesidades y expectativas de sus habitantes. Es una propuesta que permite reflexionar, sobre las características rurales, dinámicas socioculturales y visión prospectiva de su propio desarrollo, el cual, según Landini (2015) se define como un espacio en el cual, aunque hay interacciones socioculturales, le es atribuida una condición adversa, en términos de desarrollo y calidad de vida.

En este sentido, la ruralidad pasa de ser una desagregación, en donde se polariza lo urbano y lo rural a una designación de globalización, que incide en las formas en que se vinculan los territorios desde los ámbitos económicos y socioculturales, más allá de sus límites geopolíticos (Torres, 2008). Así mismo, el objetivo que propone la nueva ruralidad en América Latina muestra la complejidad de las comunidades y 
cómo estas se definen y consolidan (Carton de Grammont 2008, p. 34). El modelo para la construcción colectiva de conocimiento para la ruralidad, nacido de la intervención en el nordeste antioqueño por Instituciones de Educación Superior, identifica, a través de la interacción in situ, la forma de habitar el territorio desde lo social, económico, cultural, educativo y productivo, siendo estas interacciones, las que definen las transformaciones plurivalentes de la nueva ruralidad (Méndez, 2015).

Es así como el modelo, desde el área educativa, propende por la inclusión solidaria, un currículo flexible, experiencias didácticas creativas e innovadoras, que transforman el territorio, al igual que brinda accesibilidad, a través de las tecnologías de la información y la comunicación como elementos vinculantes a los entornos regionales, nacionales e internacionales.

\section{MATERIALES Y MÉTODOS}

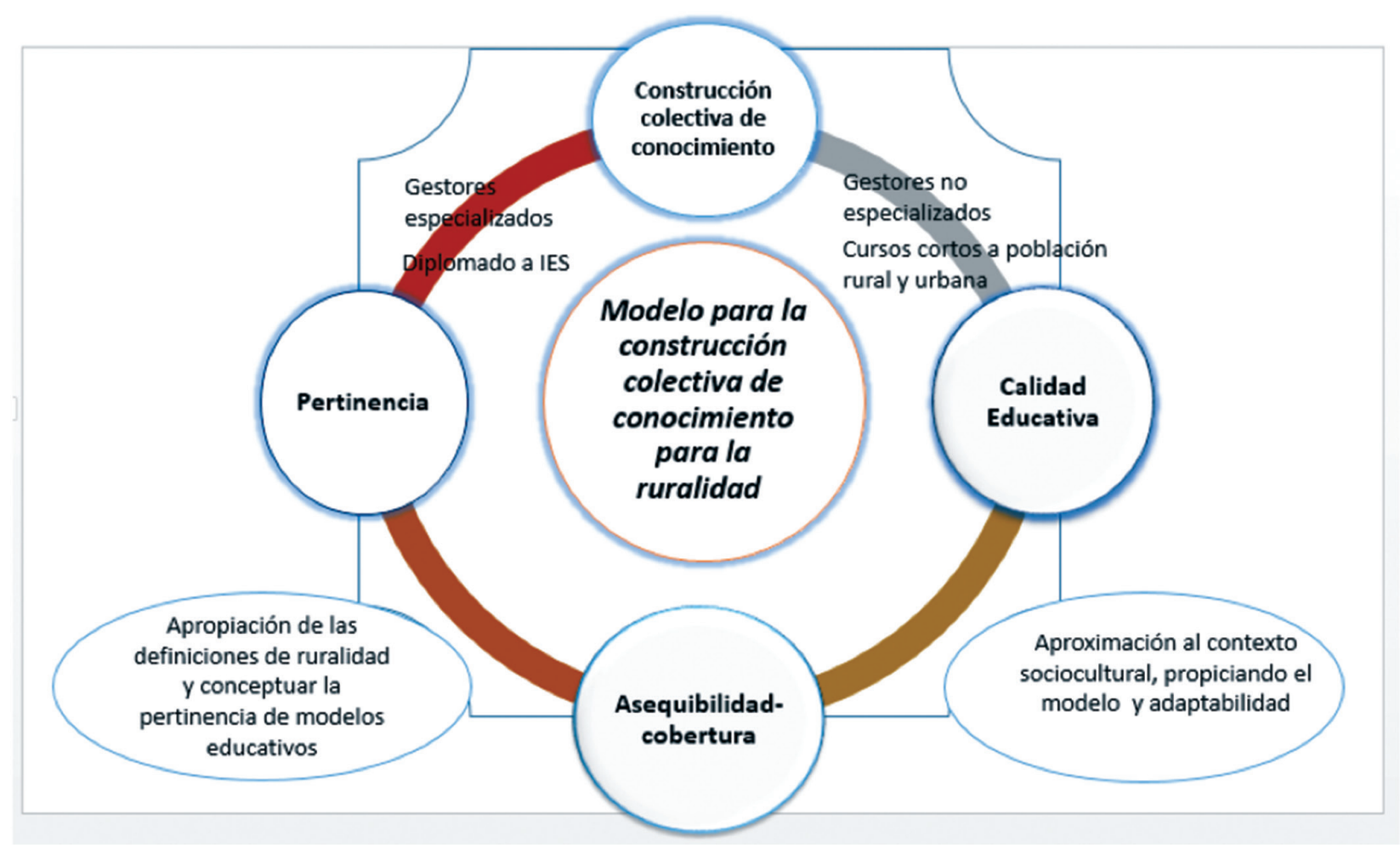

Figura 1. Contextualización metodológica.

El modelo para la construcción colectiva de conocimiento para la ruralidad se construye en las siguientes fases:1) se identifica la pertinencia de un modelo, por medio de cursos cortos a población rural y urbana; 2) Se realiza una construcción colectiva de conocimiento en la cual actúan los gestores especializados a través del diplomado Modelo colaborativo en educación superior rural y los no especializados por medio de cursos cortos con oferta diversificada en áreas académicas y funcionales; y 3) se propicia el diseño del modelo en la identificación del contexto sociocultural, desde la calidad educativa, asequibilidad-cobertura y pertinencia. 
En este mismo sentido, Malinowski (1984), explica que los métodos están conectados desde la experiencia, el conocimiento, la creatividad y la etnografía para allegarse a procesos estratégicos; es así como, mediante el método etnográfico, se interpretan las identidades y tradiciones propias de ocho (8) municipios del nordeste antioqueño (Colombia), sus niveles de desarrollo: económico, educativo, social y cultural; al igual que la participación de expertos en pedagogía, ruralidad, mediaciones digitales y modelos de educación superior, en el marco de la Organización de las Naciones Unidas para la Educación, la Ciencia y la Cultura (UNESCO), la Organización para la Cooperación y el Desarrollo Económico (OCDE) y el Ministerio de Educación Nacional (MEN), igualmente, se desarrolla la capacitación teórica institucional, se realiza la intervención in situ, en estas subregiones, mediante la cual se propicia la inquietud por el saber en sus habitantes.

En este sentido, se define el concepto de ruralidad como una hibridación de saberes y conocimiento en un contexto sociocultural que se vincula, desigualmente, a la globalidad tecnológica, económica y productiva, transformando paulatinamente la infraestructura de los territorios; es así, como, la ruralidad se caracteriza por su vocación agrícola, pecuaria, minera o turística y determina una tendencia a ejercer una u otra labor. Igualmente, influye desde su esencia (naturaleza), sobre el quehacer diario; difiere, también, en los índices de ocupación de la tierra; en este mismo sentido, se presenta una tendencia a la homogenización sociocultural, actitudinal y comportamental de los habitantes; su movilidad tiende a heredarse, su integración social es limitada y no tienen marcados condicionamientos de estratificación.

En cuanto a la globalización y su relación con los territorios rurales, la OCDE evidencia que hay un incremento en la competitividad, a la vez que se incorporan biotecnologías, biogenética, entre otras tecnologías; consecuentemente, se modifican y flexibilizan la producción y la interacción entre los sectores (OCDE, 1996).

Se seleccionan municipios geográficamente representativos del nordeste antioqueño, teniendo en cuenta la cobertura de instituciones de educación media, rurales y urbanas, interviniendo a una población de 2.627 estudiantes, habitantes de ocho (8) municipios, con la implementación de cursos cortos, a los cuales se convoca la población a través diferentes mediaciones: mixtas (presencialidad y mediación digital) y a distancia con apoyo presencial, periódico.

La Tabla 1, denominada "implementación de cursos cortos en el nordeste antioqueño", muestra las instituciones, los municipios y los estudiantes rurales y urbanos atendidos mediante cursos cortos, como parte de la aproximación a la construcción del modelo. 
Tabla 1. Implementación de cursos cortos en el nordeste antioqueño

\begin{tabular}{|c|c|c|c|}
\hline $\begin{array}{l}\text { Instituciones de educación } \\
\text { superior }\end{array}$ & $\begin{array}{l}\text { Estudiantes } \\
\text { rurales y urbanos }\end{array}$ & $\begin{array}{l}\text { Municipios } \\
\text { (número) }\end{array}$ & Municipios (nombre) \\
\hline Institución Universitaria de Envigado & 582 & 2 & Carolina del Príncipe, Amalfi, \\
\hline $\begin{array}{l}\text { Universidad de Antioquia - } \\
\text { Corporación para el fomento de } \\
\text { la educación superior - Fundación } \\
\text { Universitaria IUE }\end{array}$ & 750 & 8 & $\begin{array}{l}\text { Amalfi, Anori, Carolina del } \\
\text { príncipe, Gómez Plata, Guadalupe, } \\
\text { Remedios, Segovia, Yolombó }\end{array}$ \\
\hline $\begin{array}{l}\text { Escuela Superior Tecnológica de Artes } \\
\text { Débora Arango }\end{array}$ & 759 & 6 & $\begin{array}{l}\text { Segovia, Remedios, Gómez Plata, } \\
\text { Anorí, Amalfi, Yolombó }\end{array}$ \\
\hline Institución Universitaria Pascual Bravo & 386 & 3 & Anori, Guadalupe, Gómez Plata \\
\hline $\begin{array}{l}\text { Institución Universitaria Politécnico } \\
\text { Jaime Isaza Cadavid }\end{array}$ & 150 & 2 & Yolombó, Anori \\
\hline
\end{tabular}

Fuente: informe de ejecución de cursos cortos en el nordeste antioqueño, 2018

Con la formación teórica realizada a los participantes de las Instituciones de Educación Superior, se focaliza la acción en aspectos como los modelos pedagógicos, la política pública, la normativa estatal relacionada con la educación en el país, limitaciones y prospectiva de la educación en Colombia, y la convivencia con el contexto, condiciones y características en el nordeste antioqueño, entre otras temáticas; a través de las sesiones de formación a la población rural y urbana, se indaga, desde la lectura teórica y del contexto, los lineamientos del modelo para la construcción colectiva de conocimiento para la ruralidad.
Finalmente, se propone un modelo para la construcción colectiva de conocimiento para la ruralidad, dando respuesta a las necesidades y expectativas de los territorios del nordeste antioqueño.

\section{RESULTADOS Y DISCUSIÓN}

Colombia, en el área rural acoge el 31.6\% (Figura 1) de la población total en el país; siendo una cobertura amplia complejiza su comprensión tanto en términos de geolocalización como de desarrollo; dentro de la lógica de los últimos tiempos, lo rural se ha constituido en un problema sectorial y agropecuario, dejando una percepción de que se tiene más ruralidad que institucionalidad (PNUD, 2011) 


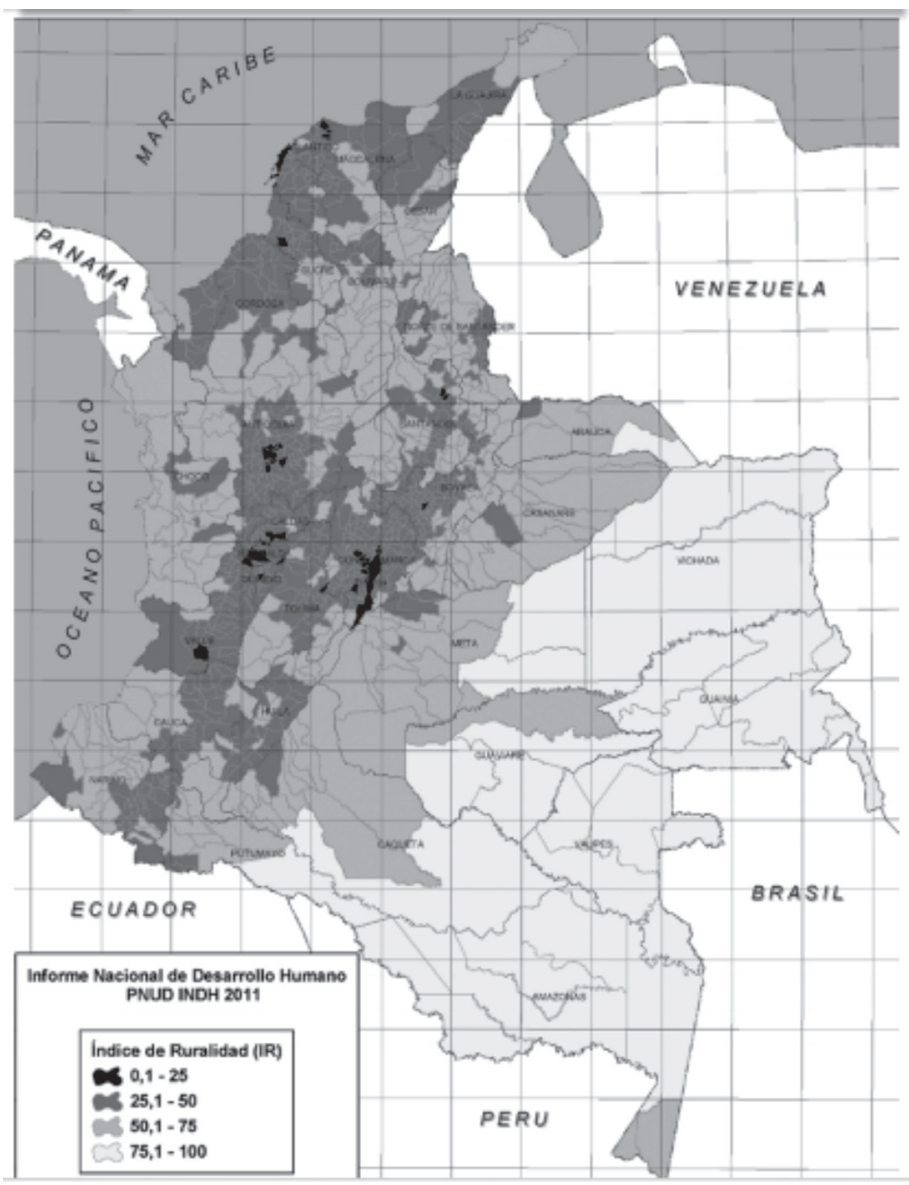

Figura 2. Mapa sobre la ruralidad en Colombia.

Fuente: Cálculos indh 2011, a partir del Censo DANE 2005.

En correlación, el informe Objetivos del Milenio (ODM), evidencia que la educación media es 2,7 veces menor que en las áreas urbanas y se exalta la necesidad de implementar y fortalecer la cobertura de esta educación media, en las áreas, identificadas como de alta ruralidad.

\section{LA OCDE Y LA EDUCACIÓN PARA LA RURALIDAD}

La OCDE (2017), en su informe sobre las Políticas Nacionales de la Educación en Colombia, expone que entre el 2000 (36\%) y el 2013 (45\%), Colombia tiene un incremento del $9 \%$ de niños matriculados, sin embargo, permanece muy por debajo del incremento promedio en los países miembros de la OCDE, con un $84 \%$; resultados a los que coadyuvan la educación gratuita, mejoramiento en la infraestructura y recursos propios y pertinentes para la educación. En éste mismo período, se presenta un incremento del $11 \%$ en las tasas netas de matriculados en la educación básica secundaria y de un $11 \%$ en la educación media, mientras que en la educación superior se incrementan en un $24 \%$ (OCDE, 2012).

Partiendo de las Políticas de calidad en la educación, en informe visibiliza los contrastes para el acceso a la educación: un estudiante ubicado en un estrato socioeconómico uno (1), solo tiene expectativa de permanecer en sus estudios por seis (6) años, mientras que un niño en estrato seis (6), podría permanecer el doble del tiempo, según la encuesta 
demográfica y de salud de Colombia (García Villegas et al., 2013; UNESCO-UIS, 2015). En éste mismo sentido, se observa que la situación socioeconómica es un factor determinante del desempeño escolar en Colombia, según las evaluaciones nacionales e internacionales (Barrera, 2014; García Villegas et al., 2013).

En conclusión, las áreas rurales (43\%) se caracterizan, por tener un índice de pobreza con un $16 \%$ mayor al índice del área urbana con un $27 \%$, (CIPI, 2013), así mismo, las comunidades indígenas y afrocolombianas, que viven en los territorios rurales, no pueden acceder a la educación (Barrera, 2014) y tampoco, accede, el $62 \%$ de los adolescentes a la educación media. En cuanto a la capacidad instalada, es insuficiente y un docente atiende entre 40 y 50 estudiantes por grupo, dificultando la formación de calidad; cuando el promedio internacional es entre 16 y 19 estudiantes por docente.

\section{COLOMBIA Y LA EDUCACIÓN PARA LA RURALIDAD}

En Colombia, según la Dirección de Fomento del MEN, el $62 \%$ de estudiantes no terminan su educación media, mientras que aproximadamente 2,5 millones, no les ha sido posible acceder a la educación universitaria. Así mismo, el Plan Nacional de Desarrollo 2014-2018, propone la disminución en la inequidad con la visión de ser el país más educado en Latinoamérica en el año 2025 (DNP, 2015) implementando políticas y estrategias, como ampliación de cobertura y mejoramiento de la calidad, jornada única, articulación de los niveles educativos, atención integral y de primera infancia, mejoramiento de la educación en la básica primaria y secundaria, incremento en el acceso y la calidad de la educación, entre otras.

Igualmente, Colombia proyecta mejorar las condiciones de calidad, cobertura y eficiencia en los procesos relacionados con la educación tanto para el área urbana como la rural. En este sentido, Herrera (2015), expone la metodología de Escuela Nueva y el Proyecto de Educación Rural (PER), las cuales han sido empleadas en la educación básica en los territorios rurales. Es así como las estructuras de base tienen un rol vital en la reconfiguración de las propuestas educativas en y para la ruralidad, teniendo presente sus características contextuales, sus necesidades y expectativas. En este sentido (Barbero, 2001) enfatiza que la articulación los conceptos subyacentes a la ruralidad con lo identitario y con las dimensiones del territorio desde lo social, lo ancestral y desde su acervo cultural.

\section{CURRÍCULO Y RESPUESTA A LOS CONTEXTOS RURALES}

Los territorios rurales tienen sus propias dinámicas que orientan el diseño de currículos satisfaciendo las necesidades y expectativas de sus habitantes, como respuesta a los problemas propios de determinado contexto, apoyando el desarrollo educativo de la región (Sepulveda \& Gallardo, 2015), lo que implica estrategias didácticas y pedagógicas, que permitan fluir una formación pertinente, resignificando saberes y conocimiento con la intención de transformar dicho territorio.

\section{MODELO PARA LA CONSTRUCCIÓN COLECTIVA DE CONOCIMIENTO EN LA RURALIDAD}

El modelo se define como la estructura sistémica, en el que las interacciones dinamizan las intencionalidades, operacionalización y autoevolución de propuestas en este caso, formativas, respondiendo a problemáticas con potenciales soluciones individuales y colaborativas.

El modelo elaborado colaborativamente, entre las instituciones de educación superior antioqueñas, tiene en cuenta los principios de: 
- Contextualización: mediante el cual se atienden las problemáticas específicas del territorio, lo que se instala el ejercicio de formación,

- Integración: como el desarrollo interdependiente entre la comunidad y su territorio,

- Flexibilidad: en una dinámica transformadora se presenta una propuesta dialógica, crítica y argumentada bajo criterios propositivos en y para la formación,

- Transversalidad: se constituye en una estrategia de aprendizaje-enseñanza o una pregunta, que se convierten en gestores de conocimiento a través de métodos o prácticas,

- y Creatividad: como respuesta al desarrollo de la propuesta formativa, tanto desde su génesis como en su desarrollo, basados en el uso de modelos pedagógicos, didácticos, medios y mediaciones digitales.

El currículo, la pedagogía y los sistemas evaluativos, como los pilares clásicos de la educación, se incorporan a las políticas regionales, nacionales e internacionales con la pretensión de resolver, en la teoría y en la práctica, los problemas que se presentan tanto desde la expectativa como desde la necesidad del contexto. En este mismo sentido, y en la era digital, el problema no es la accesibilidad a fuentes de fundamentación teórica, sino su pertinencia; esta permite conceptuar y definir con precisión la intencionalidad transformadora de sus estrategias, modelos, enfoques e interrelaciones multidireccionales.

Dentro de las políticas regionales, se plantea el incremento y la calidad en la educación para los docentes rurales, proyectos pedagógicos productivos, reducción de la brecha en la educación, ampliación de cobertura e implementación de tecnologías de la información y la comunicación integrada a la educación. La tabla 2 evidencia, la meta del plan de desarrollo del nordeste antioqueño - 2019.

Tabla 2. Metas sobre investigación y producción de conocimiento de punta

\begin{tabular}{|c|c|c|c|c|c|}
\hline $\begin{array}{c}\text { Indicador de } \\
\text { Resultado }\end{array}$ & $\begin{array}{l}\text { Nombre } \\
\text { del } \\
\text { Programa }\end{array}$ & Indicador de Producto & Unidad & $\begin{array}{c}\text { Meta del } \\
\text { Cuatrienio } \\
\text { (2016-2019) }\end{array}$ & $\begin{array}{l}\text { Dependencia } \\
\text { Responsable }\end{array}$ \\
\hline \multirow{4}{*}{$\begin{array}{l}\text { Tasa de } \\
\text { cobertura neta } \\
\text { en educación } \\
\text { media Rural }\end{array}$} & \multirow{4}{*}{$\begin{array}{l}\text { Educación } \\
\text { para la } \\
\text { nueva } \\
\text { ruralidad }\end{array}$} & $\begin{array}{l}\text { Planes de estudio ajustados a } \\
\text { la vocación productiva } \\
\text { territorial }\end{array}$ & Número & 20 - & \multirow{4}{*}{$\begin{array}{l}\text { Secretaría de } \\
\text { Educación }\end{array}$} \\
\hline & & $\begin{array}{l}\text { Programas para la formación } \\
\text { técnica, tecnológica y de } \\
\text { desarrollo humano diseñados y } \\
\text { ejecutados en la educación } \\
\text { Media Rural. }\end{array}$ & Número & 135 & \\
\hline & & 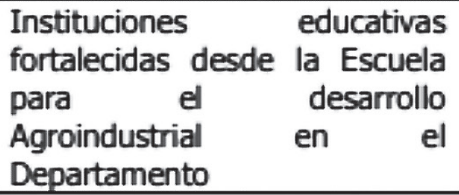 & Número & 9 & \\
\hline & & $\begin{array}{lr}\text { Proyectos } & \text { Pedagógicos } \\
\text { Productivos } & \text { (PPP) } \\
\text { implementados } & \text { con } \\
\text { estudiantes de } & \text { Instituciones } \\
\text { Educativas Rurales. } & \end{array}$ & Número & 135 & \\
\hline
\end{tabular}

Nota: En la zona rural operan 270 instituciones educativas (niveles de Preescolar-Básica y Media), 159 centros educativos rurales (hasta la Básica Secundaria) y 3.275 sedes educativas (preescolar y básica primaria, reorganizadas en una institución educativa o centro educativo). 
A partir de los antecedentes y de la integración de las Instituciones de Educación Superior para la elaboración del modelo de construcción colectiva de conocimiento para la ruralidad, y teniendo en cuenta que la realización de los cursos cortos en el nordeste convocó y cualificó 2.627 personas en áreas como inducción a la vida universitaria; ortografía para docentes y público en general; y robótica, entre otros. Es una propuesta en la que la participación cooperativa se articula en el proceso y en el desarrollo del modelo, flexibilizando creativamente desde las especificidades de cada Institución y reflexionando sobre el desarrollo del territorio, de tal modo los principios del modelo cimienten y fortalezcan el aprendizaje; en este sentido Wilson (1992) propone que

[...] la dinámica del trabajo cooperativo y colaborativo siempre estará inmersa en la formación del hombre [y de la mujer], para desarrollar y cultivar sus facultades como ser comunicativo [dialógico]...La cooperación y la colaboración están centradas en la actividad humana, como unidad mínima de ejecución con significado la cual es resultado de la acción de una persona y de su interacción con otras. Estas actividades se realizan para conseguir un objetivo usando una serie de herramientas, y todo ello, dentro de una comunidad de aprendizaje inmersa en una historia cultural. pág. 129

Desde un enfoque crítico social, valorando los aspectos socio-culturales por su carácter colaborativo, se plasma en el modelo para la construcción colectiva de conocimiento para la ruralidad, consolidando la participación y las mediaciones pertinentes de acuerdo a las posibilidades del contexto; en cuanto a la innovación, el modelo flexibiliza los procesos de formación y da cabida a la investigación y el recorrido por las fuentes de conocimiento, a lo que Monguet y Fábregas (2006) expone que:
[...] para difundir conocimiento se requiere la voluntad de un grupo o individuo para trabajar con otros y compartir conocimiento a su mutuo beneficio. Esto significa que la transferencia no ocurrirá en una organización a menos que sus integrantes y grupos de trabajo muestren un alto nivel de comportamiento cooperativo. Se encontró que las habilidades cognitivas de las personas y su disposición a cooperar determinan la eficiencia y calidad de su desempeño en las tareas que realiza en un grupo u organización. Generalmente un individuo colabora en tres niveles, de menor a mayor: Cooperación en el trabajo, difusión de los recursos (información, conocimiento y servicios), y cooperación cognitiva. En el primer nivel, los miembros de un equipo implementan sus tareas de acuerdo con el plan de tareas programadas del grupo. En el segundo nivel, los miembros del grupo se comunican para compartir información basada en un modelo de distribución definido. En el último nivel, las personas del equipo aprenden una de otra, hacen abstracciones, analogías entre problemas, y utilizan la experiencia del pasado y habilidades para resolver nuevos problemas. pág. 134

En este sentido, el aprendizaje significativo se reelabora en la medida en que se da una maduración teórica, cada vez más exigente sobre sí mismo y en relación con los grupos en que se interactúa, favoreciendo la autonomía. Es así como el modelo fundamenta la autorregulación en el proceso de formación a través de estrategias para el aprendizaje; acciones para mejorar la comprensión teórica en permanente evaluación-solución; incorporación de fortalezas psicoafectivas al currículo; identificación de focos problemáticos y sus subterfugios; y, finalmente, la elección de sistemas de seguimiento y evaluación de los procesos.

Son las condiciones del contexto las que validan el modelo desde su interacción, en la 
solución de las problemáticas, involucrando la creatividad y la autogestión. Del mismo modo, en el marco del contexto pedagógico se adscribe un grupo de docentes que trabajan estrategias aplicadas a los procesos de aprendizajeenseñanza enmarcados en los currículos. En cuanto al estudiante, propone una interacción colaborativa en su ejercicio de aprender y la producción de conocimiento se hace desde la colectividad, teniendo las mediaciones digitales como dinamizadores de su gestión y calidad.

El diseño, concibe a los sectores sociales, culturales, económicos, educativos, entre otros, como los gestores que participan y dan sostenibilidad a dicho modelo; y son los contenidos diseñados como módulos interdisciplinares e interdependientes, los que dan cuenta del diseño curricular, para la cualificación de sus habitantes y la solución de sus problemas, obteniendo una respuesta a las necesidades y expectativas que les son propias y útiles en su contexto, cuya solución se indaga teórica y contextualmente.

\section{CONCLUSIONES}

Es la permanente formación de docentes y estudiantes la que, desde una lectura de contextos, propone modelos de desarrollo para los territorios, reduciendo la brecha académica entre áreas rurales y urbanas, y dando paso al uso de las tecnologías de la información y la comunicación dentro de una globalidad, en una articulación de sinergias que propende por el cambio sostenible en la formación, tal cual lo plantea Hargreaves y Flink (2006): preservación invariante en todo aprendizaje; motivación cotidiana mediante el liderazgo y acompañamiento; una transformación en los aprendizajes; impacto en el territorio mejorando las condiciones de habitabilidad; y coherencia pedagógica mejorando los currículos y adecuándose al progresivo desarrollo de los territorios.
La construcción colectiva de los saberes, a través de los desarrollos de los gestores especializados en el diplomado Modelo colaborativo en educación superior rural y los gestores no especializados a través de los cursos cortos, proponen soluciones a las problemáticas del contexto sociocultural por medio de evaluación-solución y autogestión.

El modelo para la construcción colectiva de conocimiento para la ruralidad se caracteriza por ser inclusivo, flexible en su currículo, propende por la consolidación de experiencias didácticas, creativas e innovadoras, transformando el territorio desde una perspectiva que le es natural, propia e interdisciplinar.

\section{AGRADECIMIENTOS}

Por el compromiso institucional que facilitó el desarrollo del Modelo para la construcción colectiva de conocimiento para la ruralidad, a: institución Universitaria de Envigado, Universidad de Antioquia, Corporación para el Fomento de la Educación Superior, Fundación Universitaria IUE, Escuela Superior Tecnológica de Artes Débora Arango, Institución Universitaria Pascual Bravo, Institución Politécnica Jaime Isaza Cadavid, Empresas Públicas de Medellín.

\section{LITERATURA CITADA}

Barbero, J. M. (2001). Reconfiguraciones comunicativas de lo público. Análisis No. 26, 22-35.

Carton de Grammont, H. (2008). El concepto de nueva ruralidad. Editorial Pontificia Universidad Javeriana, (1 $1^{a}$ edición). P.p-27.

García, M, Espinosa, J, Parra, J (2017). Separados y Desiguales. Derecho, Justicia y Sociedad.

Hargreaves, A. Fink, D (2006). Estrategias de cambio y mejora en educación caracterizadas por su relevancia, difusión y continuidad en el tiempo. Revista Educación 339. 43-58.

Herrera, L, Buitrago, R. (2015). Educación Rural en Boyacá, fortalezas y debilidades desde la perspectiva del profesorado. Portal de Revistas UPTC, (4a edición). 
Landini, F. (2015). Hacia una Psicología Rural Latinoamericana. Consejo Latinoamericano de Ciencias Sociales (CLACSO).

Malinowski, B. (1984). Una Teoría Científica de La Cultura. Editorial Suramericana.

Méndez, M. (2015). Ocupación rural no agrícola y desarrollo rural local: reflexiones y aportes para una articulación efectiva. Interacciones Campo Grande, p.p-316-325.

Monguet, J. M. (2006). Efecto del blended learning sobre el rendimiento y la motivación de los estudiantes. Interciencia, pp.190-196.

OCDE. (2012). Evaluación de Políticas Nacionales de Educación: La Educación Superior en Colombia. OCDE Publishing.

Pérez, E, Farah, M, Cartón de Grammont, H. (2008). La nueva ruralidad en América
Latina. Avances teóricos y evidencias empíricas. Editorial Pontificia Universidad Javeriana p.p23-44.

PNUD. (2011). Colombia rural: Razones para la esperanza. Bogotá: INDH.

Sepúlveda, M., \& Gallardo, M. (2015). La escuela rural en la sociedad globalizada: nuevos caminos para una realidad silenciada. Profesorado: $R e-$ vista de currículum y formación del profesorado. Volumen 15, 23-45.

Torres, L. (2008). Nueva ruralidad en territorios. Consejo Nacional de Investigaciones Científicas y Tecnológicas (CONICET), p.p-201-250.

UNESCO-UIS (2015). Browse by theme: Education, Data Centre, UNESCO Institute for Statistics.

WILSON, J. D. (1992). Cómo valorar la calidad de la enseñanza. Madrid: Paidos, Iberica. 
